\title{
A Case of a Dispensing Error causing latrogenic Orthostatic Hypotension
}

By Kenneth Lam, Ann Leung, and Trevor Jaimeson

DOI: 10.22374/cjgim.v13i2.202

\section{About the Authors}

Kenneth Lam is a fellow in Geriatrics at the University of Toronto. Ann Leung is a clinical pharmacist in the cardiac ICU at St. Michael's Hospital. Trevor Jamieson is an assistant professor at the University of Toronto and a member of the Division of General Internal Medicine at St. Michael's Hospital. Corresponding author kennethk.lam@mail.utoronto.ca

Submitted: April 20, 2017; Accepted: September 19, 2017. Published: June 25, 2018.

\section{Case}

A 73-year-old man with a seven-year history of Parkinson's disease (PD) presented with delirium and increasing falls for three weeks. Past medical history included tinnitus, gout, depression, and surgically corrected carotid artery stenosis. He had a one-year history of orthostatic hypotension from PD for which he was on a stable dose of midodrine $5 \mathrm{mg}$ by mouth in the morning and noon, and $2.5 \mathrm{mg}$ by mouth in the evening. His PD was well controlled on levodopa/carbidopa. His other medications were clopidogrel, paroxetine, and rosuvastatin; there was no history of steroids. Medications had not changed recently and were administered from weekly dosettes packaged by a private caregiver. He denied cognitive changes, visual hallucinations, bleeding, nausea, vomiting, or polyuria. He had delirium.

On physical examination, profound orthostatic hypotension was noted; supine, his blood pressure was 191/93 and dropped to 119/75 mmHg when standing; his heart rate rose from 56 to 74 beats per minute. Cardiac and volume status examination were otherwise normal. Neurological examination showed masked facies but no other overt signs of PD. Cranial nerves, power, reflexes, tone, cerebellar testing, and sensation were normal.

Initial bloodwork demonstrated mild hyponatremia (131 mmol/L), hyperkalemia $(5.6 \mathrm{mmol} / \mathrm{L})$, and elevated creatinine at $219 \mu \mathrm{mol} / \mathrm{L}(110 \mu \mathrm{mol} / \mathrm{L}$ one year prior $)$. He had no anemia. Urine output was normal and bladder scan did not show retention. Computed tomography of the head showed mild cortical atrophy, and hip radiographs were negative for fracture. There were no signs of hyperpigmentation and 07:00 cortisol was $670 \mathrm{nmol} / \mathrm{L}$ (normal 193-690 nmol/L).

Initial management of the patient involved intravenous fluids and weaning paroxetine as a possible contributor to his orthostatic hypotension. In the process of collecting a best possible medication history (BPMH), the pharmacist reviewed the dosette prepared by the caregiver. The pharmacist noted that the midodrine 2.5 -mg tablets were stamped with a " 5 " instead of " 2.5 ". The caregiver remarked that the midodrine tablets had changed from round white pills to diamond-shaped pills when last dispensed 3 weeks ago. When the pharmacist reviewed an online database, she found that the patient had been supplied with 5-mg tablets of amiloride, a potassium sparing diuretic, instead of midodrine. The patient had been taking a total of $25 \mathrm{mg}$ of amiloride daily, above the maximum recommended daily dose of $20 \mathrm{mg}$.

According to the patient's pill bottle, his prescription had been for midodrine (Figure 1). We notified the community pharmacy and discovered that a dispensing error had occurred: amiloride had mistakenly been supplied instead of midodrine, having been shelved next to midodrine according to its brand name $\left(\right.$ Midamor ${ }^{\circledR}$ ). This pharmacy did not use bar-code technology.

The offending agent was stopped, and his orthostatic hypotension, electrolyte abnormalities and delirium resolved within two days. The patient was discharged in stable condition. To avoid future dispensing errors, the community pharmacy separated the two look-alike medications, held staff meetings to discuss the error, and issued a reminder for pharmacy technicians and pharmacists to both check the unique drug identification number and document this step on the dispensing record.

\section{Discussion}

By consensus, the standard definition of orthostatic hypotension is a sustained reduction of systolic blood pressure of at least $20 \mathrm{mmHg}$ or of diastolic blood pressure of $10 \mathrm{mmHg}$ within 3 


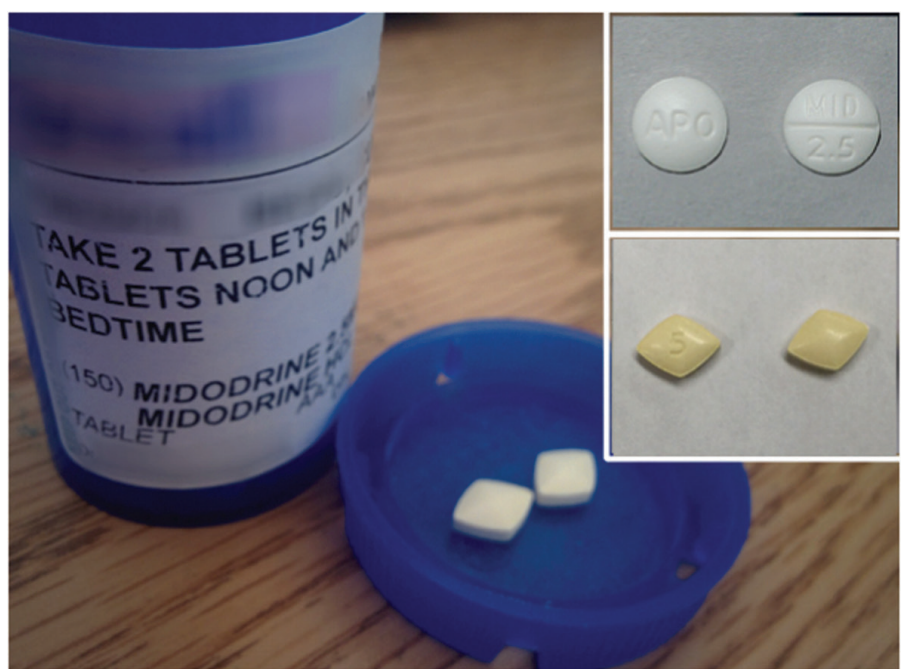

Figure 1. Image of the patient's pill bottle, labeled midodrine $2.5 \mathrm{mg}$, and the diamond-shaped pills inside, (top inset) midodrine pills for comparison, (bottom inset) diamond-shaped Midamor® pills.

minutes of standing, with or without symptoms. In 2011, the definition was revised to include several distinct variants and related conditions (Table 1$){ }^{1}$

Orthostatic hypotension is a common problem in the elderly. A longitudinal observational study of 5037 healthy adults aged 50 and over in Ireland found orthostatic hypotension occurred in $4.2 \%$ of patients aged $50-59$ and $18.5 \%$ of patients over the age of $80 .^{2}$ These results are consistent with older observational data reporting a prevalence of symptomatic orthostatic hypotension ranging between 5 and $30 \% .{ }^{3}$ Postural dizziness is not a hallmark symptom; neurologic symptoms more closely associated with orthostatic hypotension are ataxia, vertigo, dizziness and falls.

The causes of orthostatic hypotension are varied (Table $2),{ }^{5}$ and an estimated $38 \%$ of chronic cases have no identifiable cause. In this case, the relatively blunted heart rate response despite a dramatic fall in blood pressure suggested an element of autonomic dysfunction from the patient's PD. His acute renal failure with hyperkalemia and hyponatremia were more suggestive of adrenal insufficiency once urinary retention had been ruled out. Primary adrenal failure, though, was unlikely at his age and no conventional secondary etiologies were present; his morning cortisol was also well within the normal range. Volume depletion was also unlikely as he lacked polyuria and his symptoms were not fluid responsive.

The management of orthostatic hypotension involves nonpharmacologic and pharmacologic strategies, with an aim to improve symptoms and function (Table 3 ). Deprescribing potential offending agents, adequate hydration, and salt intake, and cautious transitions to a standing position are initial steps. If these fail, compression stockings, abdominal binders, and raising the head of the bed are the most effective non-pharmacological treatments based on systematic review. ${ }^{6}$ First-line pharmacotherapy includes fludrocortisone, a mineralocorticoid which promotes fluid retention and increases intravascular volume, and midodrine, a peripherally acting alpha- 1 agonist causing vasoconstriction. ${ }^{7}$ Fludrocortisone may cause hypokalemia, fluid overload, and worsen

Table 1. Definitions of Disorders of Orthostatic Tolerance

\begin{tabular}{|c|c|}
\hline Disorder & Definition \\
\hline Orthostatic hypotension & $>20 \mathrm{mmHg}$ drop in SBP or $>10 \mathrm{mmHg}$ drop in DBP within 3 minutes of standing, with or \\
\hline \multicolumn{2}{|l|}{ Variants } \\
\hline Initial orthostatic hypotension & $>40 \mathrm{mmHg}$ drop in SBP or $>20 \mathrm{mmHg}$ drop in DBP within 15 seconds \\
\hline Delayed orthostatic hypotension & Symptoms persisting beyond 3 minutes \\
\hline \multicolumn{2}{|c|}{ Related disorders of orthostatic tolerance } \\
\hline Neurally mediated syncope & Transient loss of consciousness triggered by a change in autonomic activity (e.g., cough, \\
\hline Postural tachycardia syndrome & $\begin{array}{l}\text { Increase of }>30 \text { beats per minute within } 10 \text { minutes of standing without orthostatic } \\
\text { hypotension } \\
*>40 \text { beats per minute for those aged } 12-19\end{array}$ \\
\hline
\end{tabular}

$\mathrm{DBP}=$ diastolic blood pressure; $\mathrm{SBP}=$ systolic blood pressure (SBP). 
Table 2. Common Causes of Orthostatic Hypotension

\section{Acute Causes of Orthostatic Hypotension}

Hypovolemia (e.g., dehydration, bleeding, vomiting, diarrhea)

Cardiac (e.g., arrhythmia, myocardial infarction, valvular incompetence)

Endocrine (e.g., adrenal insufficiency, hypoaldosteronism)

Medication side effects

Antihypertensives (alpha blockers, RAAS blockers, beta blockers, and diuretics)

Antipsychotics (first generation antipsychotics, clozapine, risperidone, quetiapine)

Antidepressants (tricyclic antidepressants, SNRIs, MAOIs and SSRIs)

Anticholinergics (antispasmodics)

Marijuana

Chronic Causes of Orthostatic Hypotension

Primary autonomic dysfunction

Parkinson's disease and other similar conditions (e.g., Lewy body dementia, multi-system atrophy)

Pure autonomic failure (Bradbury-Eggleston syndrome)

Secondary autonomic failure

Diabetes mellitus

Syphilis

$B_{12}$ deficiency

Amyloidosis

Paraneoplastic neuropathy

Alcoholic neuropathy

Medication side effect (see above list)

$\mathrm{MAOI}=$ monoamine oxidase inhibitors; RAAS = renin-angiotensin-aldosterone system; SNRI = serotonin and norepinephrine reuptake inhibitors; SSRI = selective serotonin reuptake inhibitors.

congestive heart failure. Midodrine has side effects of pruritis, paresthesias, piloerection and urinary retention. Both may cause supine hypertension. Second-line agents include pyridostigmine and octreotide. There is very limited evidence of the efficacy for any of these treatments as most trials of interventions are small
( $\mathrm{n}<50$ ), with no trial exceeding 200 participants, and symptoms are not always included as an outcome of interest. ${ }^{6}$ More recently, droxidopa, a norepinephrine precursor, has been approved for use in orthostatic hypotension associated with $\mathrm{PD}$ and other Parkinson-plus syndromes, but is not yet available in Canada.

Our patient's chronic orthostatic hypotension was appropriately managed with midodrine, but he suffered an adverse drug event when he was dispensed amiloride instead. Adverse drug events are estimated to be the cause of $6.5 \%$ of hospital admissions. ${ }^{8}$ Amiloride treats hypertension by blocking the epithelial sodium channel in the renal collecting duct. Orthostatic hypotension is a recognized side effect. For new admissions to hospital, screening for adverse drug events involves conducting a BPMH. Interviewing patients and caregivers to ascertain actual use of medications, including recent changes, rather than relying solely on medication lists is critical. As in this case, pharmacists have an established role in conducting medication reconciliation/ $\mathrm{BPMH},{ }^{9}$ yet it is still not usual practice to inspect and confirm the identity of the actual pills in a dosette. Attention to detail by our pharmacist, coupled with a keenly observant caregiver, allowed us to make a diagnosis that would otherwise have been missed.

The community pharmacy completed root-cause analysis internally and implemented changes to prevent similar errors from occurring in the future. The dispensing process typically involves multiple checks to ensure faithful delivery of the right drug at the right dose to the right patient with the right instructions. Dispensing errors are rarely the cause of adverse drug events; error rates vary by setting, error definition and methodology and roughly fall between 0.02 to $5 \%$ of dispensed items ${ }^{10}$. One outlier study, which used covert patients prescribed warfarin, carbamazepine or theophylline (all medications with baseline high rates of dispensing error) reported an error rate of $24 \%$, but only $4 \%$ were clinically significant. A lack of automation (e.g., bar-coding), poor lighting, interruptions, and heavy workloads likely increase the incidence of dispensing errors, ${ }^{10}$ as do look-alike drug names. Interestingly, midodrine and Midamor ${ }^{\circledast}$ do not appear on lists of look-alike drugs released by the FDA and Institute of Safe Medication Practices despite having similar dosages. This case illustrates how a dispensing error can be an occult cause of an adverse drug event and emphasizes the value of working in interdisciplinary teams as medicine becomes increasingly complex. Fortunately, this error was caught and the patient did not suffer any lasting sequelae. The feedback to the community pharmacy was well received and led to meaningful process change, highlight the importance of a culture of openly acknowledging, investigating and mitigating/preventing error as we strive for continuous quality improvement in patient safety in the health care system. 
Table 3. Non-pharmacologic and Pharmacologic Strategies For Treating Orthostatic Hypotension

\section{Non-pharmacologic Srategies}

Deprescribe offending medications

Increase fluid and salt intake

Caution with changing position from supine to standing

Compression stockings (40-60 mmHg to ankles, $30-40 \mathrm{mmHg}$ to hips)

Abdominal binders $(20-30 \mathrm{mmHg})$

Raising head of bed (by at least 6 inches)

Pharmacologic strategies

Fludrocortisone $0.1-0.3 \mathrm{mg}$ PO daily

Midodrine 2.5-10 mg PO PRN or up to 3 times daily (during hours when awake and upright)

Pyridostigmine 30-60 mg PO PRN

Octreotide 12.5-25 mcg SC PRN

Droxidopa $100 \mathrm{mg}$ PO TID, up to $1800 \mathrm{mg}$ max daily dose the postural tachycardia syndrome. Clin Auton Res 2011;21(2):6972. doi:10.1007/s10286-011-0119-5.

2. Finucane C, O'Connell MDL, Fan CW, et al. Age-Related Normative Changes in Phasic Orthostatic Blood Pressure in a Large Population Study: Findings From The Irish Longitudinal Study on Ageing (TILDA). Circulation 2014;130(20):1780-89. doi:10.1161/CIRCULATIONAHA.114.009831.

3. Low PA. Prevalence of orthostatic hypotension. Clin Auton Res 2008;18(S1):8-13. doi:10.1007/s10286-007-1001-3.

4. Rutan GH, Hermanson B, Bild DE, Kittner SJ, LaBaw F, Tell GS. Orthostatic hypotension in older adults. The Cardiovascular Health Study. CHS Collaborative Research Group. Hypertension 1992;19(6 Pt 1):508-19.

5. Gupta V, Lipsitz LA. Orthostatic Hypotension in the Elderly: Diagnosis and Treatment. Am J Med 2007;120(10):841-47. doi:10.1016/j. amjmed.2007.02.023.

6. Logan IC, Witham MD. Efficacy of treatments for orthostatic hypotension: a systematic review. Age Ageing 2012;41(5):587-94. doi:10.1093/ageing/ afs061.

7. Shibao C, Lipsitz LA, Biaggioni I, Group ASOHW. Evaluation and treatment of orthostatic hypotension. J Am Soc Hypertens 2013;7(4):317-24. doi:10.1016/j.jash.2013.04.006.

8. Pirmohamed M, James S, Meakin S, et al. Adverse drug reactions as cause of admission to hospital: prospective analysis of 18820 patients. BMJ 2004;329(7456):15-19. doi:10.1136/bmj.329.7456.15.

9. Splawski J, Minger H. Value of the Pharmacist in the Medication Reconciliation Process. P\&T 2016;41(3):176-78.

10. James KL, Barlow D, McArtney R, Hiom S, Roberts D, Whittlesea C. Incidence, type and causes of dispensing errors: a review of the literature. Int J Pharm Pract 2009;17(1):9-30. doi:10.1211/ijpp/17.1.0004.

\section{Disclosure}

The authors report no competing interests and received no funds for the creation of this article.

\section{References}

1. Freeman R, Wieling W, Axelrod FB, et al. Consensus statement on the definition of orthostatic hypotension, neurally mediated syncope and 